\title{
Digital Phenotyping Self-Monitoring Behaviors for Individuals With Type 2 Diabetes Mellitus: Observational Study Using Latent Class Growth Analysis
}

Qing Yang ${ }^{1}, \mathrm{PhD}$; Daniel Hatch ${ }^{1}, \mathrm{PhD}$; Matthew J Crowley ${ }^{2,3}$, MD; Allison A Lewinski ${ }^{2}, \mathrm{PhD}$; Jacqueline Vaughn ${ }^{1}$, BSN; Dori Steinberg ${ }^{1}, \mathrm{PhD}$; Allison Vorderstrasse ${ }^{4}, \mathrm{PhD}$; Meilin Jiang ${ }^{5}$, MSc; Ryan J Shaw ${ }^{1,6}, \mathrm{PhD}$

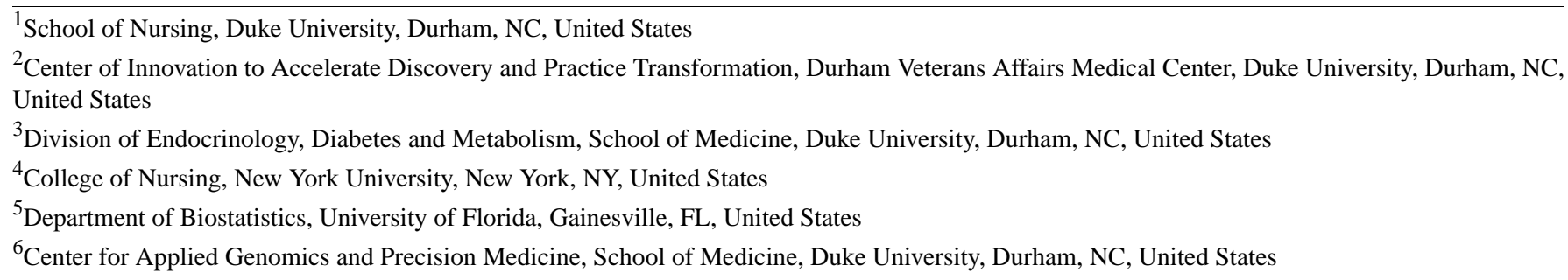

\section{Corresponding Author:}

Qing Yang, PhD

School of Nursing

Duke University

307 Trent Drive

Durham, NC, 27710

United States

Phone: 19196139768

Email: qing.yang@duke.edu

\section{Abstract}

Background: Sustained self-monitoring and self-management behaviors are crucial to maintain optimal health for individuals with type 2 diabetes mellitus (T2DM). As smartphones and mobile health (mHealth) devices become widely available, self-monitoring using mHealth devices is an appealing strategy in support of successful self-management of T2DM. However, research indicates that engagement with mHealth devices decreases over time. Thus, it is important to understand engagement trajectories to provide varying levels of support that can improve self-monitoring and self-management behaviors.

Objective: The aims of this study were to develop (1) digital phenotypes of the self-monitoring behaviors of patients with T2DM based on their engagement trajectory of using multiple mHealth devices, and (2) assess the association of individual digital phenotypes of self-monitoring behaviors with baseline demographic and clinical characteristics.

Methods: This longitudinal observational feasibility study included 60 participants with T2DM who were instructed to monitor their weight, blood glucose, and physical activity using a wireless weight scale, phone-tethered glucometer, and accelerometer, respectively, over 6 months. We used latent class growth analysis (LCGA) with multitrajectory modeling to associate the digital phenotypes of participants' self-monitoring behaviors based on their engagement trajectories with multiple mHealth devices. Associations between individual characteristics and digital phenotypes on participants' self-monitoring behavior were assessed by analysis of variance or the Chi square test.

Results: The engagement with accelerometers to monitor daily physical activities was consistently high for all participants over time. Three distinct digital phenotypes were identified based on participants' engagement with the wireless weight scale and glucometer: (1) low and waning engagement group (24/60, 40\%), (2) medium engagement group (20/60,33\%), and (3) consistently high engagement group $(16 / 60,27 \%)$. Participants that were younger, female, nonwhite, had a low income, and with a higher baseline hemoglobin $A_{1 c}$ level were more likely to be in the low and waning engagement group.

Conclusions: We demonstrated how to digitally phenotype individuals' self-monitoring behavior based on their engagement trajectory with multiple mHealth devices. Distinct self-monitoring behavior groups were identified. Individual demographic and clinical characteristics were associated with different self-monitoring behavior groups. Future research should identify methods to provide tailored support for people with T2DM to help them better monitor and manage their condition. 
International Registered Report Identifier (IRRID)： RR2-10.2196/13517

(JMIR Mhealth Uhealth 2020;8(6):e17730) doi: $\underline{10.2196 / 17730}$

\section{KEYWORDS}

digital phenotype; latent class growth analysis; type 2 diabetes; self-management; self-monitoring; Mobile Health

\section{Introduction}

Sustained self-management with consistent self-monitoring is essential for individuals with type 2 diabetes mellitus (T2DM) to help them maintain optimal health [1]. Mobile health (mHealth) devices (eg, apps, Fitbit, Apple Watch, wireless scale, glucometer) are widely available and can help support engagement in T2DM self-management behaviors [2]. Using mHealth devices to monitor weight, blood glucose levels, activity levels, and dietary behaviors has proven to be feasible and effective in adults with T2DM [3-5]. Despite these benefits of mHealth tools, research indicates that engagement with mHealth tools decreases over time, and these trends also vary according to individual characteristics [6-10]. Determining these patterns of engagement with mHealth tools over time and how individual characteristics are associated with various patterns may provide crucial understanding on the use of mHealth tools to support T2DM self-monitoring and self-management.

Digital phenotyping, the concept of using data from mHealth devices to augment assessment of human illness, is rapidly emerging [11]. To date, digital phenotyping has been successfully used to track behavior and symptom data and to refine diagnosis and risk prediction for psychiatric disorders [12], dementia [13], and asthma [14]. Digital phenotyping has also been used to facilitate chronic disease management, such as using wearable accelerometers to track functional outcomes in patients with neurological disorders and to facilitate rehabilitation programs [15]. Although the actual readings or values from mHealth devices provide vital information for disease diagnosis, prognosis, and management, the engagement trajectories with multiple mHealth devices over time also provide crucial information about self-monitoring behaviors for patients with chronic diseases. Individuals who engage with mHealth devices more frequently indicate better self-monitoring behavior.

Latent class growth analysis (LCGA) is a type of growth mixture model that can determine individual phenotypes by identifying subgroups who follow similar trajectories over time on one or more outcomes. LCGA has been used extensively in the social sciences [16]. In medical and nursing research, LCGA has been used to quantify patient risk profiles based on physiological measures [17] and symptom research [18]. The method was extended by Jones and Nagin [19] to identify distinct subgroups based on trajectories across multiple outcomes.

In this study, we sought to demonstrate how to digitally phenotype the self-monitoring behaviors of individuals with T2DM based on their engagement trajectories with multiple mHealth devices using LCGA with multitrajectory modeling. Further, we explored if the participants' digital phenotypes on self-monitoring behaviors varied according to their baseline demographic and clinical characteristics.

\section{Methods}

\section{Design and Sample}

This was a longitudinal observational feasibility study using multiple mHealth devices for patients with T2DM. The complete details of the study protocol were previously reported [5]. Sixty individuals with T2DM were recruited from a single primary care clinic and were followed for 6 months. As described in Table 1, participants were asked to perform self-monitoring using three measures on three mobile devices provided by the study over 6 months. This included weight (pounds) measured by a cellular-enabled scale provided by BodyTrace (Palo Alto, CA, USA), blood glucose $(\mathrm{mg} / \mathrm{dL})$ measured through a phone-tethered glucometer provided by iHealth (Mountain View, CA, USA), and physical activity measured in daily steps by a wrist-worn accelerometer and associated fitness app provided by Fitbit (San Francisco, CA, USA). Participants reported demographic information at baseline in Research Electronic Data Capture, a secure, web-based software platform designed to support data capture for research studies [20,21]. Daily monitoring on weight and physical activity were required by the study protocol, whereas blood glucose monitoring was prescribed by the primary care physician, which was performed at least once a week. Participants' hemoglobin $\mathrm{A}_{1 \mathrm{c}}\left(\mathrm{HbA}_{1 \mathrm{c}}\right)$ values were extracted from their electronic health record from the closest date to baseline and 6 months postbaseline. Duke University's Institutional Review Board approved all study activities (IRB No. Pro00071569).

Table 1. Mobile health devices used and time points for data collection.

\begin{tabular}{lll}
\hline Variable & Instrument & Time points \\
\hline Weight (pounds) & Cellular-enabled Scale (BodyTrace) & Daily \\
Blood glucose (mg/dL) & $\begin{array}{l}\text { Food and Drug Association-approved wireless glucometer } \\
\text { (iHealth) }\end{array}$ & $\begin{array}{l}\text { As prescribed by the primary care physician, } \\
\text { at least once a week }\end{array}$ \\
Physical activity (number of steps) & Triaxial accelerometer and associated fitness app (Fitbit) & Daily \\
Hemoglobin $\mathrm{A}_{1 \mathrm{c}}(\mathrm{mmol} / \mathrm{mol})$ & Electronic health record laboratory results & Baseline and 6 months postbaseline
\end{tabular}




\section{Measures}

Self-monitoring behaviors were captured by engagement with different mHealth devices on tracking weight, blood glucose, and physical activity. We operationalized engagement with the wireless weight scale and accelerometer as the percentage of days that the participants used the devices during 13 biweekly periods over 6 months. Since some participants may measure blood glucose multiple times per day, we operationalized engagement with the glucometer as the percentage of days that participants measured blood glucose at least once a day

Covariates included age (years), gender, race (nonwhite, white), income $(1=<\mathrm{US} \$ 10,000, \quad 2=\mathrm{US} \quad \$ 10,000-19,999, \quad 3=\mathrm{US}$ $\$ 20,000-29,999,4=U S \$ 30,000-39,999,5=U S \$ 40,000-49,999$, $6=$ US $\$ 50,000-59,999,7=$ US $\$ 60,000-79,999,8 \geq U S \$ 80,000$ ), insulin dependence (currently taking any insulin medication), and baseline $\mathrm{HbA}_{1 \mathrm{c}}$ values.

\section{Data Analysis}

Descriptive statistics were used to summarize demographic and other participant characteristics at baseline, including age, gender, race, income, insulin dependence, and $\mathrm{HbA}_{1 \mathrm{c}}$ level. Empirical summary plots of biweekly engagement rates over 6 months were created for each device to illustrate the trajectories of self-monitoring behaviors for weight, blood glucose, and physical activity.

We conducted LCGA using SAS Proc Traj [19,22] to identify latent groups of trajectories in biweekly engagement over the 6-month observation period. We first modeled the trajectories of biweekly engagement of each device separately to determine the number of latent classes that offered the best fit for each device. Because engagement rate is a continuous variable with an approximately normal distribution, we used the censored normal distribution (cnorm). Based on the empirical summary plots, we tested both linear and quadratic trend models and chose the number of latent groups based on different number of groups using both the Akaike information criterion (AIC) and Bayesian information criterion (BIC) values in addition to clinical judgement of the study team. After modeling the trajectories for each device, we modeled the trajectories of adherence to the devices simultaneously using a multitrajectory model.

To examine the relationships between participant characteristics, clinical variables, and latent trajectory group membership, we conducted analysis of variance (ANOVA) for age, income level, and baseline $\mathrm{HbA}_{1 \mathrm{c}}$ value, and Chi square tests for race, gender, and insulin dependence. Finally, ANOVA was conducted to assess if a latent trajectory group identified in the LCGA multitrajectory model was associated with 6-month $\mathrm{HbA}_{1 \mathrm{c}}$ values and changes in $\mathrm{HbA}_{1 \mathrm{c}}$ from baseline to 6 months. All data analyses were conducted with SAS 9.4 (Cary, NC, USA).

\section{Results}

\section{Sample}

Demographic characteristics are presented in Table 2. The majority of the participants were women and nonwhite. The median income was US $\$ 40,000-49,999$, and approximately half of the participants were currently using insulin medication. More detailed information on the sample and recruitment was reported previously [10].

Table 2. Demographic characteristics $(\mathrm{N}=60)$.

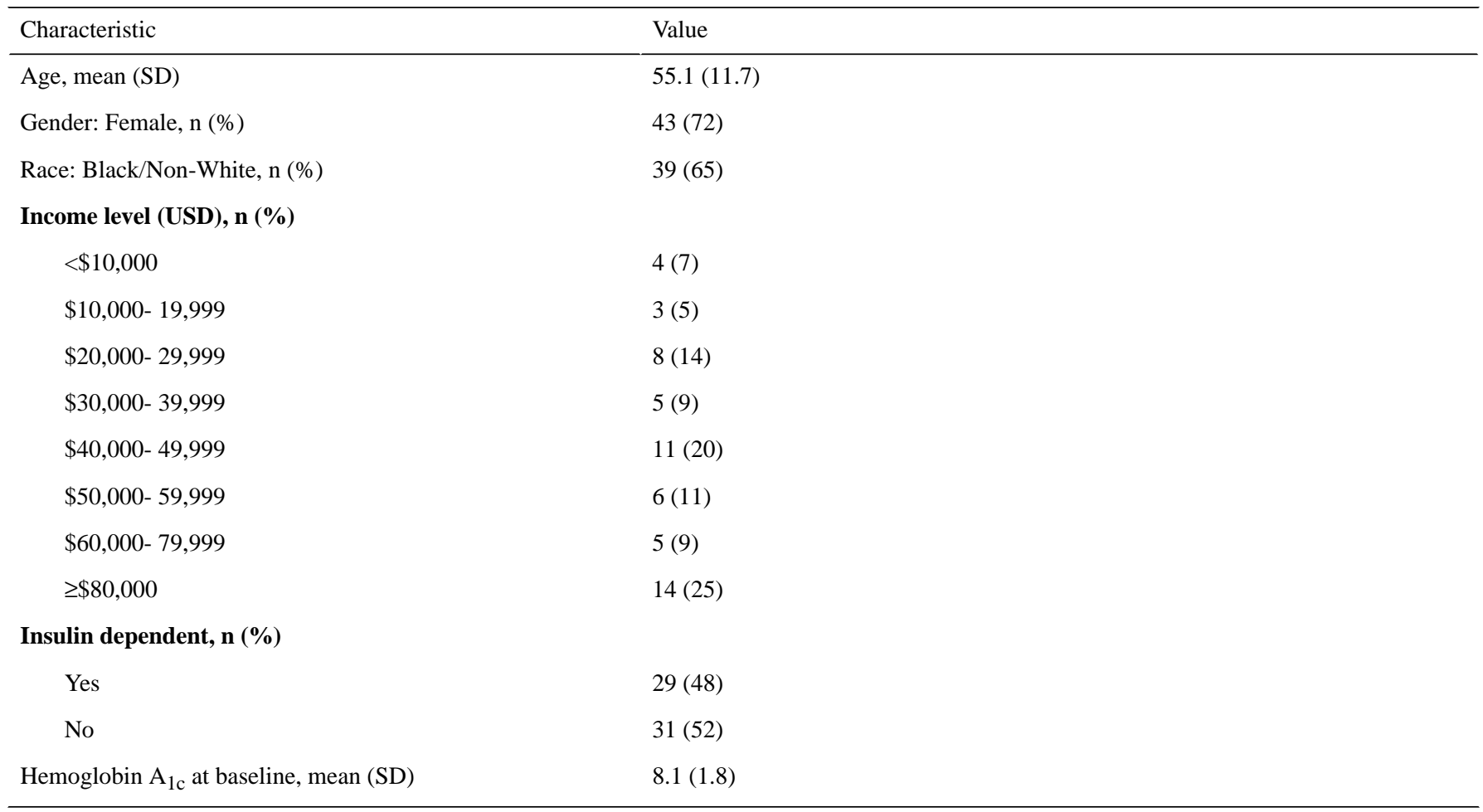




\section{Engagement Trajectories and Self-Monitoring Behavior Phenotypes}

Empirical summary plots of engagement with different mHealth devices are presented in Figure 1 to show overall engagement trends. The average engagements with the wireless scale and glucometer for all participants were moderate (52\%-72\%) and showed a slightly decreasing trend for the first 2 weeks. In contrast, engagements with the accelerometers remained high over time at around $90 \%$ and with very minimal variability across all participants.

Three latent classes of engagement trajectories were identified (Table 3, Figure 2): low and waning engagement group, medium engagement group, and consistently high engagement group. The AIC and BIC values of this model were -825.1 and -846.0, respectively. In the low engagement group, individuals had relatively lower engagement with daily weight and glucose monitoring at baseline (40\% and 56\%, respectively) and showed a statistically significant decrease in daily weight and glucose monitoring over time. The drop in engagement with daily weight monitoring was faster in the first 2 weeks and was captured by a significant quadratic term. The moderate engagement group showed moderate engagement with daily weight and glucose monitoring at baseline (65\% and $72 \%$, respectively) and no statistically significant change over time. In the high engagement group, high engagement with daily weight and glucose monitoring at baseline was observed $(82 \%$ and $94 \%$, respectively). In this group, a slight but statistically significant increase in weight monitoring was observed over the 6 months, whereas glucose monitoring did not change. The final three-class model was chosen based on a model fit procedure according to AIC and BIC values (Table 4). For all devices, model fit was improved in the three-class model compared to the two-class model. However, for weight and glucose, fit improved only marginally in the four-class model relative to the three-class model. For physical activity, the four-class model could not be produced. The final three-class models were based on weight and glucose device engagement trajectories because the engagement rate for the physical activity device was consistently high over time for all participants with very small variabilities.

Figure 1. Empirical plots (mean, SEM) for biweekly engagement trajectories for each mobile health device over all 6 months.

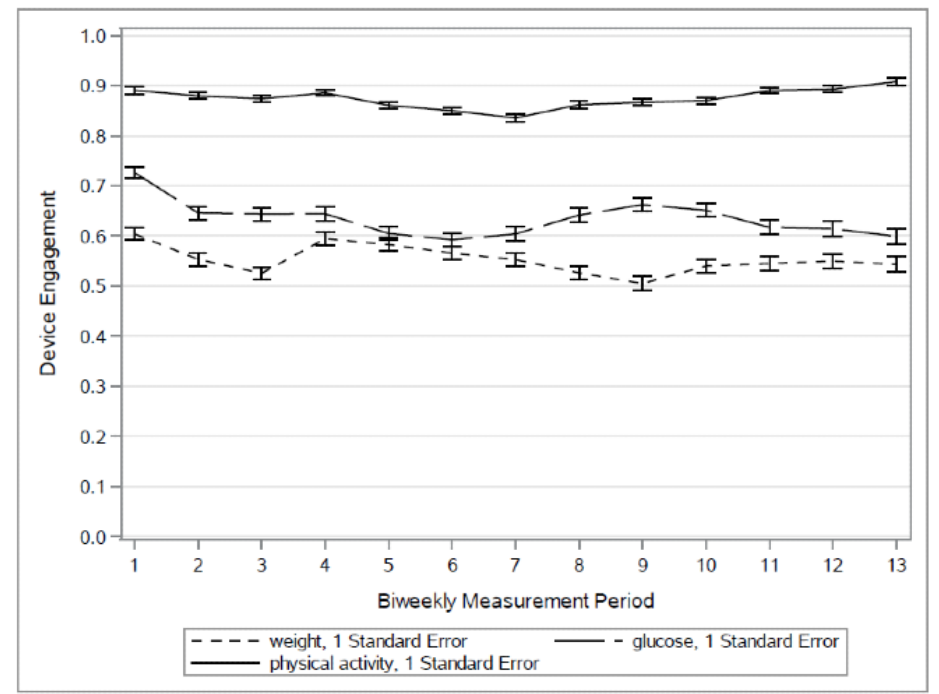


Table 3. Latent class growth analysis multitrajectory model results for engagement with a wireless weight scale and glucometer $(\mathrm{N}=60)$.

\begin{tabular}{|c|c|c|c|c|}
\hline Variable & B & SE & $t_{1}$ & $P$ value \\
\hline \multicolumn{5}{|l|}{ Group membership } \\
\hline Low Engagement $(\mathrm{n}=24)$ & $40 \%$ & 6.40 & 6.21 & $<.001$ \\
\hline Medium Engagement $(\mathrm{n}=20)$ & $33 \%$ & 6.30 & 5.27 & $<.001$ \\
\hline High Engagement ( $\mathrm{n}=16)$ & $27 \%$ & 5.91 & 4.57 & $<.001$ \\
\hline \multicolumn{5}{|l|}{ Weight } \\
\hline \multicolumn{5}{|l|}{ Low Engagement } \\
\hline linear & -0.09 & 0.02 & -3.67 & $<.001$ \\
\hline quadratic & 0.005 & 0.002 & 2.82 & .005 \\
\hline \multicolumn{5}{|l|}{ Medium Engagement } \\
\hline linear & 0.02 & 0.03 & 0.71 & .48 \\
\hline quadratic & -0.003 & 0.002 & -1.41 & .16 \\
\hline \multicolumn{5}{|l|}{ High Engagement } \\
\hline linear & 0.02 & 0.008 & 2.34 & .02 \\
\hline \multicolumn{5}{|l|}{ Glucose } \\
\hline \multicolumn{5}{|l|}{ Low Engagement } \\
\hline linear & -0.07 & 0.03 & -2.27 & .02 \\
\hline quadratic & 0.002 & 0.002 & 0.90 & .37 \\
\hline \multicolumn{5}{|l|}{ Medium Engagement } \\
\hline linear & -0.02 & 0.03 & -0.62 & .54 \\
\hline quadratic & 0.001 & 0.002 & 0.60 & .55 \\
\hline \multicolumn{5}{|l|}{ High Engagement } \\
\hline linear & -0.003 & 0.01 & -0.27 & .79 \\
\hline
\end{tabular}

Figure 2. Empirical summary plot for biweekly engagement trajectories with the (A) glucometer and (B) wireless weight scale by different engagement groups.
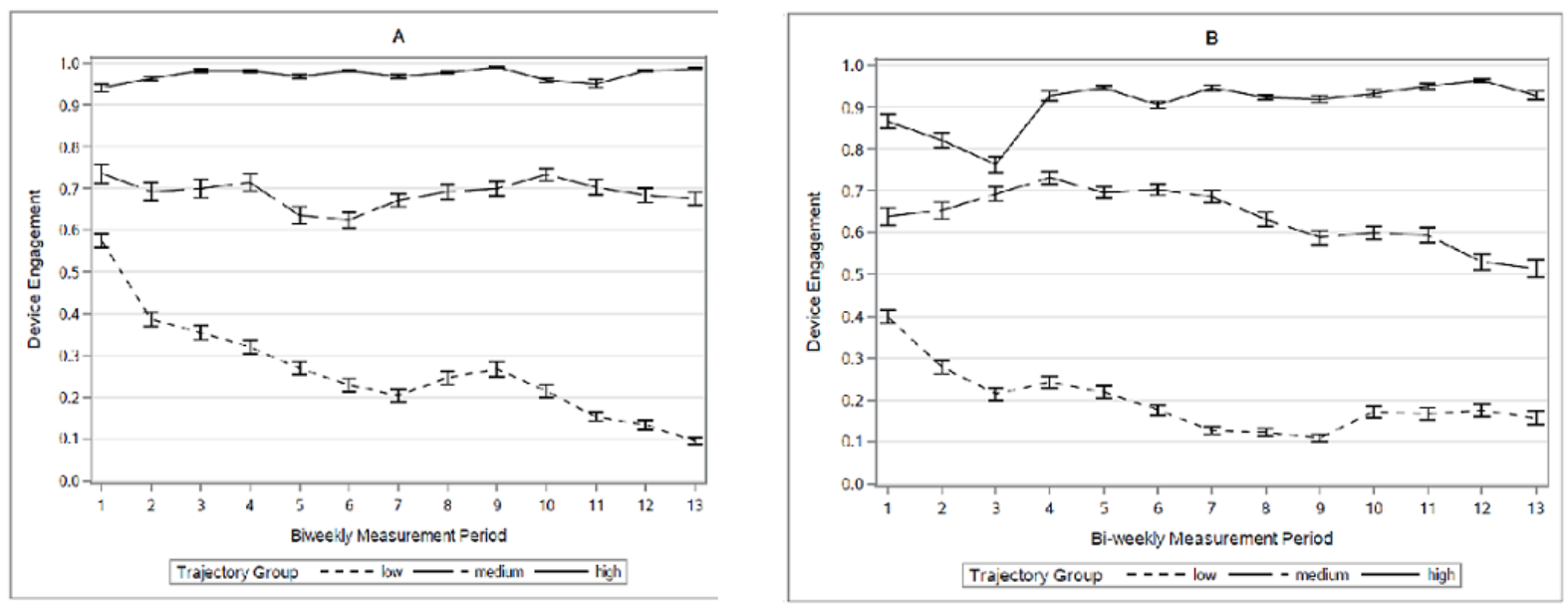
Table 4. Model fit by number of latent classes modeleda.

\begin{tabular}{|c|c|c|c|c|c|c|}
\hline \multirow[t]{2}{*}{ Number of classes } & \multicolumn{3}{|l|}{ Weight } & \multicolumn{3}{|c|}{ Glucose } \\
\hline & $\mathrm{AIC}^{\mathrm{b}}$ & $\mathrm{BIC}^{\mathrm{c}}$ & Percent per class & AIC & $\mathrm{BIC}$ & Percent per class \\
\hline 2 & -420.1 & -427.4 & $48.7 / 51.3$ & -457.4 & -464.7 & $56.3 / 43.7$ \\
\hline 3 & -368.4 & -379.9 & $39.3 / 20.8 / 39.9$ & -408.3 & -419.8 & $45.1 / 25.0 / 29.8$ \\
\hline 4 & -352.2 & -368.0 & $8.7 / 32.4 / 19.1 / 39.7$ & -393.3 & -409.0 & 29.0/16.7/24.4/29.9 \\
\hline
\end{tabular}

${ }^{\mathrm{a}}$ Sample size per class is based on most likely class membership.

${ }^{\mathrm{b}} \mathrm{AIC}$ : Akaike information criterion

${ }^{\mathrm{c}} \mathrm{BIC}$ : Bayesian information criterion.

\section{Associations of Self-Monitoring Behavior Phenotypes With Participant and Clinical Characteristics}

The results of bivariate analyses examining how self-monitoring phenotypes are related to participant characteristic are presented in Table 5. Self-monitoring phenotype was significantly associated with age, in that those in the low and waning engagement group were younger compared to those in the medium and consistently high engagement groups. Self-monitoring phenotype was also significantly related to gender as there were less women in the high and medium engagement groups compared to the low engagement group. Race was also significantly associated with group membership, in that those in the low engagement group were more likely to be nonwhite than those in the high engagement group. Insulin dependence was not significantly associated with engagement group.

Table 6 summarizes the association between the self-monitoring behavior phenotypes and $\mathrm{HbA}_{1 \mathrm{c}}$ at baseline, 6 months, and the change between baseline and 6 months. Although the change in $\mathrm{HbA}_{1 \mathrm{c}}$ from baseline to 6 months did not differ according to engagement group, the low engagement group had higher baseline $\mathrm{HbA}_{1 \mathrm{c}}$ values compared to those of the medium and high engagement groups. This trend continued at the 6-month follow up, with higher $\mathrm{HbA}_{1 \mathrm{c}}$ values in the low engagement group compared to the medium and high engagement groups.

Table 5. Bivariate relationships between baseline demographic and clinical characteristics and engagement group membership.

\begin{tabular}{|c|c|c|c|c|c|}
\hline Variable & $\begin{array}{l}\text { Low Engagement } \\
(24 / 60,40 \%)\end{array}$ & $\begin{array}{l}\text { Medium Engagement } \\
(20 / 60,33 \%)\end{array}$ & $\begin{array}{l}\text { High Engagement } \\
(16 / 60,27 \%)\end{array}$ & Test statistic & $P$ value \\
\hline Age, mean (SD) & $49.1(13.0)$ & $57.9(10.5)$ & $60.6(6.3)$ & $F_{2,57}=6.48$ & .003 \\
\hline $\begin{array}{l}\text { Gender: Female, } \mathrm{n} \\
(\%)\end{array}$ & $20(47)$ & $10(23)$ & $13(30)$ & $\chi^{2}{ }_{2}=6.96$ & .03 \\
\hline $\begin{array}{l}\text { Income }^{\mathrm{a}} \text {, mean } \\
\text { (SD) }\end{array}$ & $4.3(2.2)$ & $5.7(2.0)$ & $5.9(2.3)$ & $F_{2,53}=3.13$ & .05 \\
\hline $\begin{array}{l}\text { Race: Black/Non- } \\
\text { White, n (\%) }\end{array}$ & $20(51)$ & $11(28)$ & $8(21)$ & $\chi_{2}^{2}=6.01$ & .05 \\
\hline Insulin dependent, n (\%) & & & & $\chi_{2}^{2}=1.80$ & .41 \\
\hline Yes & $14(48)$ & $9(31)$ & $6(21)$ & & \\
\hline No & $10(33)$ & $11(36)$ & $10(32)$ & & \\
\hline $\begin{array}{l}\text { Hemoglobin } A_{1 c} \text { at } \\
\text { baseline, mean } \\
\text { (SD) }\end{array}$ & $9.01(2.13)$ & $7.61(1.17)$ & $7.34(1.16)$ & $F_{2,56}=6.30$ & .003 \\
\hline
\end{tabular}

${ }^{\mathrm{a}}$ Income categories: $1=<\$ 10,000,2=\$ 10,000-19,999,3=\$ 20,000-29,999,4=\$ 30,000-39,999,5=\$ 40,000-49,999 ', 6=\$ 50,000-59,999,7=\$ 60,000-79,999$, $8 \geq \$ 80,000$.

Table 6. Hemoglobin $\mathrm{A}_{1 \mathrm{c}}$ levels (mean, $\mathrm{SD}$ ) at baseline, 6 months, and change according to multitrajectory engagement group (N=60).

\begin{tabular}{|c|c|c|c|c|c|c|}
\hline Time point & Low Engagement $(n=24)$ & Medium Engagement $(n=20)$ & High Engagement ( $\mathrm{n}=16)$ & $F$ & df & $P$ value \\
\hline Baseline & $9.01(2.13)$ & $7.61(1.17)$ & $7.34(1.16)$ & 6.30 & 2,56 & .003 \\
\hline Six months & $8.64(2.54)$ & $7.09(1.45)$ & $7.16(1.23)$ & 3.96 & 2,49 & .03 \\
\hline $\begin{array}{l}\text { Change from baseline to } 6 \\
\text { months }\end{array}$ & $0.0(2.23)$ & $-0.44(1.07)$ & $-0.19(0.64)$ & 0.38 & 2,48 & .68 \\
\hline
\end{tabular}




\section{Discussion}

Consistent self-monitoring and self-management of T2DM improves health outcomes [1,23,24]. Given the growing popularity of using mHealth devices to facilitate self-monitoring, engagement with mHealth devices has become an important tool to develop digital phenotypes based on individuals' self-monitoring behaviors. This study is among the first to operationalize digital phenotyping of self-monitoring behaviors by applying LCGA modeling on engagement trajectory data from multiple mHealth devices.

Overall, individuals' engagement with an accelerometer to monitor daily physical activities was consistently high $(>82 \%)$ for the participants over time. Similar patterns were observed in other mHealth studies $[25,26]$. This may be due to the passive nature of data collection and transmission of these devices. We were able to identify three distinct digital phenotypes of self-monitoring behaviors using engagement trajectories of wireless weight scales and glucometers. There was a low and waning engagement group $(24 / 60,40 \%)$, a medium engagement over time group $(20 / 60,33 \%)$, and a consistently high engagement group $(16 / 60,27 \%)$. Specifically, the low engagement group started with low engagement in using both the wireless scale (40\%) and glucometer (58\%), and then the level of engagement rapidly decreased in the first 2 weeks.

These results are similar with those of other studies focused on the use of mHealth technologies or devices for chronic disease management [7]. However, our study provides further evidence by identifying individuals with low engagement in the first couple weeks, demonstrating the need to allocate additional intervention or resources since it is likely that waning engagement will be observed over time. For people who are highly engaged initially, we could consider providing minimum support to save resources as they will be more likely to stay engaged over time.

Our findings also demonstrate how engagement with mHealth devices varies according to patient demographic and clinical characteristics. The individuals in the high and moderate engagement groups were older, included more men, had higher income levels, were more likely to be white, and had better $\mathrm{HbA}_{1 \mathrm{c}}$ values at baseline. By contrast, the low and waning engagement group members were younger, included more women, had lower incomes, were more likely to self-identify as black or nonwhite, and had poorer control of their T2DM. Participants who are insulin-dependent may be required to self-monitor their blood glucose daily or even multiple times a day based on instructions from their primary care physician. This will certainly increase the motivation to engage with the glucometer or even wireless scales for the study participants. However, we did not find any significant association between insulin dependence and engagement group. This implies that we may need to provide further support to this high-risk group. The baseline characteristics of our sample are similar to those of prior research in that lower income individuals, nonwhite individuals, and women face more challenges in controlling glycemia, experience more T2DM complications, and have higher mortality rates $[6,27]$. We hypothesize that the younger patients in our study may have had lower engagement due to competing demands on their time (eg, caring for family, work), more comorbidities, or having been diagnosed with diabetes at a younger age. Digital phenotypes of self-monitoring behaviors can identify patients who may need the most support in changing health care behaviors and can inform strategies to tailor the use of mHealth tools in the delivery of self-management interventions. This result also indicates that different retention approaches may be needed for certain populations to maintain engagement with mHealth tools in support of T2DM self-management.

As discussed above, individuals with well-controlled baseline $\mathrm{HbA}_{1 \mathrm{c}}$ were more likely to be in the consistently high engagement group. Not surprisingly, these individuals continued to have better controlled $\mathrm{HbA}_{1 \mathrm{c}}$ at the 6-month follow up. However, the change in $\mathrm{HbA}_{1 \mathrm{c}}$ value between baseline and the 6-month follow up did not differ according to different phenotypes of self-monitoring behaviors or engagement groups. This indicates that good self-monitoring behaviors through active engagement with mHealth devices is helpful in maintaining well-controlled $\mathrm{HbA}_{1 \mathrm{c}}$, but does not necessarily further reduce $\mathrm{HbA}_{1 \mathrm{c}}$.

Limitations to the study include that the sample was obtained from a single site in the southeastern United States, which may not be representative of all patients with T2DM. A larger-scale study that includes more patients from different regions would yield more generalizable findings to a broader population. Such a study would also help to identify more complex patterns in engagement trajectories and more specific strategies in delivering behavior change interventions. Self-monitoring also occurred for only 6 months, which did not allow for examination of long-term patterns in a complex chronic illness such as T2DM. There are several factors that may affect a patient's motivation to engage with the device and self-monitoring that was not accounted for in our analysis. First, this was an observational study and the participants were provided with different mHealth devices, which they could keep if they completed the study. Although we did not have any specific requirement or incentive for participants to use the device during the follow-up time, the ability to keep the device may have some implications in retaining their participation in the study. Second, during the 6-month follow-up period of the study, we conducted 20 interviews with the participants to view their data and gain perspectives on using real-time data collections to support self-monitoring. This may have also potentially affected the motivation for participants to engage.

In conclusion, T2DM is a challenging disease that requires frequent self-monitoring and consistent self-management. Digital phenotyping on self-monitoring behaviors using LCGA can help to identify subgroups of individuals with distinct engagement trajectories. Future research should focus on methods to develop tailored mHealth interventions based on the influence of different phenotypes of individuals on their self-monitoring behaviors. 


\section{Acknowledgments}

This study was funded by a grant from the US National Institutes of Health, the National Institute of Nursing Research (NINR 1R15NR015890 to RS, QY, DS, AV, MC), a Duke University Center for Nursing Research Pilot grant (QY), and a Duke University Data+ grant (RS). This study was also supported by the Building Interdisciplinary Careers in Women's Health Career Development Award (K12HD043446 to DS); US Department of Veterans Affairs Office of Academic Affiliations (grant No. TPH 21-000 to AL); Durham Center of Innovation to Accelerate Discovery and Practice Transformation (Grant No. CIN 13-410 to MC and AL); and the US National Institutes of Health (NINR F31NR018100 to JV). The content is solely the responsibility of the authors and does not necessarily reflect the position or policy of Duke University, the US Department of Veterans Affairs, or the US government. The authors acknowledge iHealth for donation of devices and would like to thank Angel Barnes, the study coordinator, for her dedication to assure the accuracy of the data, and Judith C. Hays and Karen Judge for editorial assistance.

\section{Conflicts of Interest}

DS is a consultant with Omada Health. The other authors declare no conflict of interest.

\section{References}

1. Powers MA, Bardsley J, Cypress M, Duker P, Funnell MM, Fischl AH, et al. Diabetes Self-Management Education and Support in Type 2 Diabetes: A Joint Position Statement of the American Diabetes Association, the American Association of Diabetes Educators, and the Academy of Nutrition and Dietetics. J Acad Nutr Diet 2015 Aug;115(8):1323-1334. [doi: 10.1016/j.jand.2015.05.012] [Medline: 26054423]

2. Shaw RJ, Bonnet JP, Modarai F, George A, Shahsahebi M. Mobile health technology for personalized primary care medicine. Am J Med 2015 Jun;128(6):555-557. [doi: 10.1016/j.amjmed.2015.01.005] [Medline: 25613298]

3. Greenwood DA, Gee PM, Fatkin KJ, Peeples M. A Systematic Review of Reviews Evaluating Technology-Enabled Diabetes Self-Management Education and Support. J Diabetes Sci Technol 2017 Sep;11(5):1015-1027 [FREE Full text] [doi: 10.1177/1932296817713506] [Medline: 28560898]

4. Wu X, Guo X, Zhang Z. The Efficacy of Mobile Phone Apps for Lifestyle Modification in Diabetes: Systematic Review and Meta-Analysis. JMIR Mhealth Uhealth 2019 Jan 15;7(1):e12297 [FREE Full text] [doi: 10.2196/12297] [Medline: 30664494]

5. Shaw RJ, Barnes A, Steinberg D, Vaughn J, Diane A, Levine E, et al. Enhancing Diabetes Self-Management Through Collection and Visualization of Data From Multiple Mobile Health Technologies: Protocol for a Development and Feasibility Trial. JMIR Res Protoc 2019 Jun 03;8(6):e13517 [FREE Full text] [doi: $\underline{10.2196 / 13517]}$ [Medline: $\underline{31162127}$ ]

6. Milward J, Deluca P, Drummond C, Kimergård A. Developing Typologies of User Engagement With the BRANCH Alcohol-Harm Reduction Smartphone App: Qualitative Study. JMIR Mhealth Uhealth 2018 Dec 13;6(12):e11692 [FREE Full text] [doi: 10.2196/11692] [Medline: $\underline{\text { 30545806] }}$

7. Goh G, Tan NC, Malhotra R, Padmanabhan U, Barbier S, Allen JC, et al. Short-term trajectories of use of a caloric-monitoring mobile phone app among patients with type 2 diabetes mellitus in a primary care setting. J Med Internet Res 2015;17(2):e33 [FREE Full text] [doi: 10.2196/jmir.3938] [Medline: 25648130]

8. Pham Q, Graham G, Carrion C, Morita PP, Seto E, Stinson JN, et al. A Library of Analytic Indicators to Evaluate Effective Engagement with Consumer mHealth Apps for Chronic Conditions: Scoping Review. JMIR Mhealth Uhealth 2019 Jan 18;7(1):e11941 [FREE Full text] [doi: 10.2196/11941] [Medline: 30664463]

9. Koot D, Goh PSC, Lim RSM, Tian Y, Yau TY, Tan NC, et al. A Mobile Lifestyle Management Program (GlycoLeap) for People With Type 2 Diabetes: Single-Arm Feasibility Study. JMIR Mhealth Uhealth 2019 May 24;7(5):e12965 [REE Full text] [doi: 10.2196/12965] [Medline: $\underline{31127720]}$

10. Shaw RJ, Yang Q, Barnes A, Hatch D, Crowley MJ, Vorderstrasse A, et al. Self-monitoring diabetes with multiple mobile health devices. J Am Med Inform Assoc 2020 May 01;27(5):667-676. [doi: 10.1093/jamia/ocaa007] [Medline: 32134447]

11. Jain SH, Powers BW, Hawkins JB, Brownstein JS. The digital phenotype. Nat Biotechnol 2015 May;33(5):462-463. [doi: 10.1038/nbt.3223] [Medline: 25965751]

12. Torous J, Kiang MV, Lorme J, Onnela J. New Tools for New Research in Psychiatry: A Scalable and Customizable Platform to Empower Data Driven Smartphone Research. JMIR Ment Health 2016 May 05;3(2):e16 [FREE Full text] [doi: 10.2196/mental.5165] [Medline: 27150677]

13. Ienca M, Vayena E, Blasimme A. Big Data and Dementia: Charting the Route Ahead for Research, Ethics, and Policy. Front Med (Lausanne) 2018;5:13. [doi: 10.3389/fmed.2018.00013] [Medline: 29468161]

14. Jaimini U, Thirunarayan K, Kalra M, Venkataraman R, Kadariya D, Sheth A. "How Is My Child's Asthma?" Digital Phenotype and Actionable Insights for Pediatric Asthma. JMIR Pediatr Parent 2018;1(2):e11988 [FREE Full text] [doi: 10.2196/11988] [Medline: $\underline{31008446]}$

15. Steins D, Dawes H, Esser P, Collett J. Wearable accelerometry-based technology capable of assessing functional activities in neurological populations in community settings: a systematic review. J Neuroeng Rehabil 2014 Mar 13;11:36 [FREE Full text] [doi: $\underline{10.1186 / 1743-0003-11-36}$ ] [Medline: $\underline{\text { 24625308] }}$ 
16. Jung T, Wickrama KAS. An Introduction to Latent Class Growth Analysis and Growth Mixture Modeling. Social Pers Psych Compass 2008 Jan;2(1):302-317. [doi: 10.1111/j.1751-9004.2007.00054.x]

17. Allen NB, Siddique J, Wilkins JT, Shay C, Lewis CE, Goff DC, et al. Blood pressure trajectories in early adulthood and subclinical atherosclerosis in middle age. JAMA 2014 Feb 05;311(5):490-497 [FREE Full text] [doi: 10.1001/jama.2013.285122] [Medline: 24496536]

18. Hockenberry MJ, Hooke MC, Rodgers C, Taylor O, Koerner KM, Mitby P, et al. Symptom Trajectories in Children Receiving Treatment for Leukemia: A Latent Class Growth Analysis With Multitrajectory Modeling. J Pain Symptom Manage 2017 Jul;54(1):1-8 [ [FREE Full text] [doi: 10.1016/j.jpainsymman.2017.03.002] [Medline: 28433546]

19. Jones BL, Nagin DS. Advances in Group-Based Trajectory Modeling and an SAS Procedure for Estimating Them. Sociol Methods Res 2016 Jun 30;35(4):542-571. [doi: 10.1177/0049124106292364]

20. Harris PA, Taylor R, Minor BL, Elliott V, Fernandez M, O'Neal L, REDCap Consortium. The REDCap consortium: Building an international community of software platform partners. J Biomed Inform 2019 Jul;95:103208. [doi: 10.1016/j.jbi.2019.103208] [Medline: 31078660]

21. Harris PA, Taylor R, Thielke R, Payne J, Gonzalez N, Conde JG. Research electronic data capture (REDCap)--a metadata-driven methodology and workflow process for providing translational research informatics support. J Biomed Inform 2009 Apr;42(2):377-381 [FREE Full text] [doi: 10.1016/j.jbi.2008.08.010] [Medline: 18929686]

22. Jones BL, Nagin DS. A Note on a Stata Plugin for Estimating Group-based Trajectory Models. Sociol Methods Res 2013 Sep 30;42(4):608-613. [doi: 10.1177/0049124113503141]

23. Hartz J, Yingling L, Powell-Wiley TM. Use of Mobile Health Technology in the Prevention and Management of Diabetes Mellitus. Curr Cardiol Rep 2016 Dec;18(12):130. [doi: 10.1007/s11886-016-0796-8] [Medline: 27826901]

24. Steinsbekk A, Rygg L, Lisulo M, Rise MB, Fretheim A. Group based diabetes self-management education compared to routine treatment for people with type 2 diabetes mellitus. A systematic review with meta-analysis. BMC Health Serv Res 2012 Jul 23;12:213 [FREE Full text] [doi: 10.1186/1472-6963-12-213] [Medline: 22824531]

25. Janevic MR, Shute V, Murphy SL, Piette JD. Acceptability and Effects of Commercially Available Activity Trackers for Chronic Pain Management Among Older African American Adults. Pain Med 2020 Feb 01;21(2):e68-e78. [doi: 10.1093/pm/pnz215] [Medline: $\underline{\text { 31509196] }}$

26. Mendoza JA, Baker KS, Moreno MA, Whitlock K, Abbey-Lambertz M, Waite A, et al. A Fitbit and Facebook mHealth intervention for promoting physical activity among adolescent and young adult childhood cancer survivors: A pilot study. Pediatr Blood Cancer 2017 Dec;64(12). [doi: 10.1002/pbc.26660] [Medline: 28618158]

27. Glazier RH, Bajcar J, Kennie NR, Willson K. A systematic review of interventions to improve diabetes care in socially disadvantaged populations. Diabetes Care 2006 Jul;29(7):1675-1688. [doi: 10.2337/dc05-1942] [Medline: 16801602]

\section{Abbreviations \\ AIC: Akaike information criterion \\ ANOVA: analysis of variance \\ BIC: Bayesian information criterion \\ $\mathbf{H b A}_{1 \mathbf{c}}$ : hemoglobin $\mathrm{A}_{1 \mathrm{c}}$ \\ LCGA: latent class growth analysis \\ mHealth: mobile health \\ T2DM: type 2 diabetes mellitus}

Edited by G Eysenbach; submitted 08.01.20; peer-reviewed by H Mehdizadeh, T Powell-Wiley; comments to author 21.03.20; revised
version received 30.03.20; accepted 31.03.20; published 11.06.20
Please cite as:
Yang Q, Hatch D, Crowley MJ, Lewinski AA, Vaughn J, Steinberg D, Vorderstrasse A, Jiang M, Shaw RJ
Digital Phenotyping Self-Monitoring Behaviors for Individuals With Type 2 Diabetes Mellitus: Observational Study Using Latent
Class Growth Analysis
JMIR Mhealth Uhealth 2020;8(6):e17730
URL: $\underline{\text { https:///mhealth.jmir.org/2020/6/e17730 }}$
doi: $10.2196 / 17730$
PMID: $\underline{32525492}$

(C) Qing Yang, Daniel Hatch, Matthew J Crowley, Allison A Lewinski, Jacqueline Vaughn, Dori Steinberg, Allison Vorderstrasse, Meilin Jiang, Ryan J Shaw. Originally published in JMIR mHealth and uHealth (http://mhealth.jmir.org), 11.06.2020. This is an open-access article distributed under the terms of the Creative Commons Attribution License 
(https://creativecommons.org/licenses/by/4.0/), which permits unrestricted use, distribution, and reproduction in any medium, provided the original work, first published in JMIR mHealth and uHealth, is properly cited. The complete bibliographic information, a link to the original publication on http://mhealth.jmir.org/, as well as this copyright and license information must be included. 